\title{
Dynamics of WIMPs in the Solar System and Implications for Direct and Indirect Detection
}

\author{
Annika H. G. Peter* \\ Department of Physics, Princeton University, Princeton, NJ 08544 \\ Caltech MS 105-24, Pasadena, CA 91125 \\ apeter@astro.caltech.edu

\section{Scott Tremaine} \\ Department of Astrophysical Sciences, Princeton University, Princeton, NJ 08544 \\ School of Natural Sciences, Institute for Advanced Study, Princeton, NJ 08540 \\ tremainedias.edu.
}

\begin{abstract}
Semi-analytic treatments of the evolution of orbits of weakly interacting massive particles (WIMPs) in the solar system suggest that the WIMPs bound to the solar system may enhance the direct detection rate relative to that of the unbound population by up to a factor of order unity, and boost the flux of neutrinos from WIMP annihilation in the Earth by up to two orders of magnitude. To test these important but uncertain results, we perform a suite of numerical orbit integrations to explore the properties of the bound WIMP population as a function of the WIMP mass and the scattering cross section with baryonic matter. For regions of WIMP parameter space presently allowed by experiments, we find that (i) the bound WIMP population enhances the direct detection rate by at most $\sim 1 \%$ relative to the rate from unbound halo WIMPs; (ii) it is unlikely that planned $\mathrm{km}^{3}$-scale neutrino telescopes will detect neutrinos from WIMP annihilation in the Earth; (iii) the event rate from neutrinos produced by WIMP annihilation in the Sun may be much smaller than implied by the usual calculations, which assume that WIMPs scattered onto bound orbits are rapidly thermalized in the Sun.
\end{abstract}

Identification of dark matter 2008

August 18-22, 2008

Stockholm, Sweden

${ }^{*}$ Speaker. 


\section{Introduction}

WIMPs in the solar system may be detected in (i) "direct detection" experiments, which measure the recoil of nuclei during interactions with astrophysical WIMPs [1] and have placed interesting constraints on the WIMP mass $m_{\chi}$ and spin-independent (or -dependent) elastic scattering cross section with protons $\sigma_{p}^{S I}$ and neutrons $\sigma_{n}^{S I}\left(\sigma_{p}^{S D}\right.$ and $\left.\sigma_{n}^{S D}\right)$; and (ii) "indirect detection" experiments, including neutrino telescopes. The next generation of neutrino telescopes may detect the high-energy neutrinos from annihilation of WIMPs captured in the Sun and Earth [2].

For a given WIMP model, event rates in direct and indirect detection experiments are determined by the phase-space distribution function (DF) of WIMPs. The fiducial assumption is that these event rates are dominated by WIMPs from the Galactic halo, passing through the solar system on unbound orbits [⿰氵⿱一𫝀]. . However, the following two processes may create additional long-lived populations of WIMPs bound to the solar system. (i) Damour \& Krauss [4] argued that secular gravitational interactions with the planets could increase the perihelia of Galactic WIMPs scattered in the Sun to larger radii, quenching further scattering by solar nuclei and dramatically increasing the WIMP lifetimes. Their semi-analytic estimates indicated that the bound population could increase direct detection rates by up to a factor of $\mathscr{O}(1)$, and enhance the neutrino flux from the Earth by two orders of magnitude [4]. (ii) Gould and Lundberg \& Edsjö [5] estimated that the density of bound WIMPs at Earth due to gravitational capture and scattering by the planets was $\sim 1 \%$ of the density of unbound WIMPs if additional elastic scattering in the Sun were unimportant. Because of their low geocentric speeds the bound WIMPs could enhance the capture rate in the Earth by up to an order of magnitude, hence boosting the neutrino flux.

Semi-analytic techniques cannot, however, describe the full spectrum of behavior in few-body gravitational systems like the solar system. Numerical orbit integrations are the only reliable tool for this task. In this paper, we summarize the results of a set of such integrations, which are described further in [6].

\section{Simulations}

We assume that the DF of the halo dark matter is Maxwellian, with a local halo WIMP number density $n_{\chi}=\rho_{\chi} / m_{\chi} . m_{\chi}$ is the WIMP mass, and we set the local WIMP mass density $\rho_{\chi}=$ $0.3 \mathrm{GeV} \mathrm{cm}^{-3}$. We set the speed of the Sun relative to the halo to $v_{\odot}=220 \mathrm{~km} \mathrm{~s}^{-1}$, and the onedimensional WIMP velocity dispersion $\sigma=v_{\odot} / \sqrt{ } 2$.

The initial conditions for the orbit integration are of two kinds. Type I: We employ Monte Carlo methods to determine the positions and velocities of bound WIMPs after they have scattered elastically off nuclei in the Sun (using the solar model in [7). We run four sets of simulations, each corresponding to a particular choice of WIMP spin-independent elastic scattering cross section and mass $\left(\sigma_{p}^{S I}=10^{-41} \mathrm{~cm}^{2}: m_{\chi}=60\right.$ AMU ; $\sigma_{p}^{S I}=10^{-43} \mathrm{~cm}^{2}: m_{\chi}=60,150$ and 500 AMU $)$. The spin-dependent cross section is set to zero for simplicity. Type II: To estimate the DF of WIMPs captured gravitationally, WIMP orbits are followed numerically after they approach within 1000 AU of the Sun. We carry out the simulations with only a single mass and cross section $\left(m_{\chi}=500\right.$ AMU, $\sigma_{p}^{S I}=10^{-43} \mathrm{~cm}^{2}$ ), but record the integrated optical depth from passages through the Sun as a function of time, which allows us to extend the results to other masses and cross sections. 


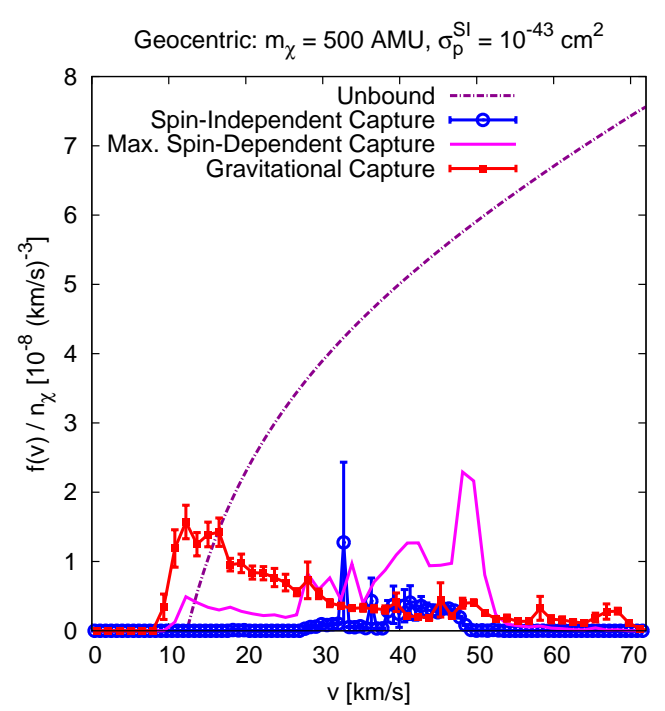

Figure 1: DFs as a function of geocentric speed $v$, normalized so that $\int f(v) v^{2} d v$ is the number density; $n_{\chi}$ is the halo number density in the solar neighborhood. The dashed line is the unbound halo DF. The circles represent Type I initial conditions with $\sigma_{p}^{S D}=0$, the squares show the bound DF from gravitational capture (Type II), and the solid line without points is the estimated DF for Type I capture with $\sigma_{p}^{S D}=10^{-36} \mathrm{~cm}^{2}$.

Bound WIMPs are followed numerically, admitting the possibility of additional elastic scattering in the Sun. We use a simplified solar system containing only one planet, Jupiter, revolving on a circular orbit about the Sun. The integration algorithm is symplectic with an adaptive timestep determined by the heliocentric distance [8]. We use other methods to integrate orbits when WIMPs go through the Sun or have close encounters with planets. Integration is terminated before the age of the solar system if the WIMP escapes from the solar system or is scattered in the Sun onto an orbit with semi-major axis $a<0.5 \mathrm{AU}$. Type I simulations followed $10^{5}-10^{6}$ particles. Type II simulations followed over $10^{10}$ particles, of which $\sim 3 \times 10^{5}$ were at least temporarily bound.

\section{Results}

We present a sample of our results in Fig. 1, which shows DFs $f(v)$ at the Earth as a function of geocentric speed $v$, in the case of $m_{\chi}=500 \mathrm{AMU}$ and $\sigma_{p}^{S I}=10^{-43} \mathrm{~cm}^{2}$. The dashed line denotes WIMPs from the halo, unbound to the solar system. Blue circles show the DF of WIMPs with Type I initial conditions with $\sigma_{p}^{S D}=0$. Red squares represent the WIMPs with Type II initial conditions. Errors are estimated using bootstrap resampling. We extrapolate our results to obtain a maximum DF due to spin-dependent scattering (the solid magenta line). The main conclusion in Fig. 1 is that the total bound number density is small, no more than $\sim 0.1 \%$ of the unbound density.

We estimate the direct detection rates of bound WIMPs, assuming that spin-independent interactions dominate in the detector volume. The analysis thresholds of most experiments lie above the maximum possible recoil energy for bound WIMPs $(<10 \mathrm{keV})$. An exception is the XENON10 experiment [1]], for which the maximum enhancement to the differential event rate is $\sim 1 \%$ of the halo event rate. The enhancement to the total event rate in the analysis window is $\sim 0.1 \%$. 
We determine event rates in neutrino telescopes from WIMP annihilation in the Earth using the DarkSUSY software package, assuming the WIMP is a supersymmetric [9]. We find that the event rate in an IceCube-sized neutrino telescope [2] is below the detection threshold.

The Sun is a target for WIMP annihilation searches. Initial results from the Super-Kamiokande experiment suggest that neutrino telescopes will be competitive with direct detection experiments in their sensitivity to $\sigma_{p}^{S D}$, with the standard assumption of rapid thermalization of WIMPs in the Sun [10]. The neutrino flux from the Sun is usually estimated assuming that all particles scattered in the Sun onto bound orbits rapidly reach thermal equilibrium with solar nuclei, settling into a dense core at the center of the Sun if $m_{\chi} \gtrsim 4 \mathrm{Gev}$ [11]. Thermalization is crucial since the annihilation rate goes as the square of the density. This picture may be realistic if $m_{\chi} \lesssim 1 \mathrm{TeV}$. However, we find that most WIMPs on Jupiter-crossing orbits are ejected from the solar system before they can rescatter in the Sun (so long as $\sigma_{p}^{S D} \lesssim 10^{-38} \mathrm{~cm}^{2}$ ). There are also long-lived WIMP populations that may not thermalize. We find that if $\sigma_{p}^{S D} \lesssim 10^{-43} \mathrm{~cm}^{2}\left(\gtrsim 10^{-43} \mathrm{~cm}^{2}\right)$ and $m_{\chi}=1 \mathrm{TeV}, 20 \%$ (12\%) of WIMPs will never thermalize in the Sun, and rises to near $100 \%(85 \%)$ for $m_{\chi}=10 \mathrm{TeV}$.

\section{Acknowledgments}

We thank A. Serenelli for providing the standard solar model in tabular form. We acknowledge financial support from NASA grants NNG04GL47G and NNX08AH24G. The simulations were run on computers at Princeton University supported by the Departments of Astrophysical Sciences (NSF AST-0216105) and Physics, and the TIGRESS High Performance Computing Center.

\section{References}

[1] UK Dark Matter Collaboration, Phys. Lett. B 616, 17 (2005), D. S. Akerib et al. (CDMS), Phys. Rev. D 73, 011102 (2006); H. S. Lee et al. (KIMS), Phys. Rev. Lett. 99, 091301 (2007); E. Behnke et al. (COUPP), Science 319, 933 (2008); J. Angle et al. (XENON10), Phys. Rev. Lett. 100, 021303 (2008); CDMS Collaboration (2008), arXiv:0802.3530.

[2] P. Amram et al., Nucl. Phys. B Proc. Suppl. 75, 415 (1999); G. C. Hill et al. (2006), arXiv:astro-ph/0611773; C. de los Heros et al. (2008), arXiv:0802.0147.

[3] G. Jungman, M. Kamionkowski, and K. Griest, Phys. Rep. 267, 195 (1996); H.-C. Cheng, J. L. Feng, and K. T. Matchev, Phys. Rev. Lett. 89, 211301 (2002); G. Servant and T. M. P. Tait, New J. Phys. 4, 99 (2002); J. Hubisz and P. Meade, Phys. Rev. D 71, 035016 (2005).

[4] T. Damour and L. M. Krauss, Phys. Rev. D 59, 063509 (1999); L. Bergström et al., JHEP 8, 10 (1999).

[5] A. Gould, ApJ 368, 610 (1991); J. Lundberg and J. Edsjö, Phys. Rev. D 69, 123505 (2004).

[6] A. H. G. Peter (2008), in prep.; Ph.D. thesis, Princeton University.

[7] J. N. Bahcall, A. M. Serenelli, and S. Basu, ApJ 621, L85 (2005).

[8] S. Mikkola and K. Tanikawa, Cel. Mech. Dyn. Astron. 74, 287 (1999); M. Preto and S. Tremaine, AJ 118, 2532 (1999).

[9] P. Gondolo et al., JCAP 7, 8 (2004).

[10] S. Desai et al., Phys. Rev. D 70, 083523 (2004).

[11] T. K.Gaisser, G. Steigman, and S. Tilav, Phys. Rev. D 34, 2206 (1986). 